\title{
Lack of immunoediting in murine pancreatic cancer reversed with neoantigen
}

\author{
Rebecca A. Evans, Mark S. Diamond, Andrew J. Rech, Timothy Chao, Max W. Richardson, \\ Jeffrey H. Lin, David L. Bajor, Katelyn T. Byrne, Ben Z. Stanger, James L. Riley, Nune Markosyan, \\ Rafael Winograd, and Robert H. Vonderheide
}

Abramson Family Cancer Research Institute, Perelman School of Medicine, University of Pennsylvania, Philadelphia, Pennsylvania, USA.

\begin{abstract}
In carcinogen-driven cancers, a high mutational burden results in neoepitopes that can be recognized immunologically. Such carcinogen-induced tumors may evade this immune response through "immunoediting," whereby tumors adapt to immune pressure and escape T cell-mediated killing. Many tumors lack a high neoepitope burden, and it remains unclear whether immunoediting occurs in such cases. Here, we evaluated T cell immunity in an autochthonous mouse model of pancreatic cancer and found a low mutational burden, absence of predicted neoepitopes derived from tumor mutations, and resistance to checkpoint immunotherapy. Spontaneous tumor progression was identical in the presence or absence of $\mathrm{T}$ cells. Moreover, tumors arising in $\mathrm{T}$ cell-depleted mice grew unchecked in immune-competent hosts. However, introduction of the neoantigen ovalbumin (OVA) led to tumor rejection and $\mathrm{T}$ cell memory, but this did not occur in OVA immune-tolerant mice. Thus, immunoediting does not occur in this mouse model - a likely consequence, not a cause, of absent neoepitopes. Because many human tumors also have a low missense mutational load and minimal neoepitope burden, our findings have clinical implications for the design of immunotherapy for patients with such tumors.
\end{abstract}

Conflict of interest: The authors have declared that no conflict of interest exists.

Submitted: April 29, 2016 Accepted: July 28, 2016 Published: September 8, 2016

Reference information: JCI Insight. 2016;1(14):e88328. doi:10.1172/jci.insight.88328.

\section{Introduction}

Interactions between $\mathrm{T}$ cells and cancer cells are considered a critical component of cancer biology and can be successfully exploited to treat patients (1). As demonstrated in landmark studies of highly immunogenic tumors in mouse models, such as the methylcholanthrene (MCA) tumor model (2-4), tumors can avoid T cell destruction if tumors are "edited" under the Darwinian-like pressure exerted by tumor-specific $\mathrm{T}$ cells or, alternatively, if tumors induce $\mathrm{T}$ cell tolerance or mediate immunosuppression. These tumors progress despite tumor $\mathrm{T}$ cell infiltration (5). However, there is also evidence that certain murine tumors grow independently of $\mathrm{T}$ and $\mathrm{B}$ cells $(6-9)$, with a histological picture of immune privilege $(10,11)$ and few intratumoral $\mathrm{T}$ cells. It remains unclear whether immunoediting also plays a role in these tumors. Features of the tumor and tumor microenvironment that dictate the development of immunoediting versus other mechanisms of immunosurveillance are not fully understood.

We therefore reassessed cardinal features of cancer immunosurveillance using a genetically engineered mouse model (GEMM) of pancreatic ductal adenocarcinoma (PDA) in which spontaneous, nonimmunogenic tumors arise and mimic the human disease and tumor microenvironment with high fidelity $(12,13)$ We designed an experimental approach to mimic the classic immune surveillance experiments performed in the MCA model but used the "KPC" model instead. In the KPC model of PDA, targeted pancreatic expression of mutant Kras and p53 at the endogenous loci drives tumorigenesis in immune-competent hosts without exposure to carcinogens (14). The KPC model is an important tool to study human PDA, which is highly lethal, almost universally driven by mutant Kras, and predicted to become the second leading cause of cancer death in the United States by 2020 (15). PDA in both humans and KPC mice is characterized by a dense, desmoplastic stroma, which features a prominent network of immunosuppressive leukocytes driven in part by the tumor itself (12). Most importantly, this cancer represents a class of solid tumors that has remained largely refractory to checkpoint immunotherapy. Tumors in the KPC model fail 
to regress with anti-PD-1 or anti-CTLA-4 $(16,17)$. In KPC mice, antitumor T cell immunity is barely measurable (17) and effector $\mathrm{T}$ cell infiltration into tumors is minimal at even the earliest stage of neoplasia $(12,13,18)$ - similar to the dearth of $\mathrm{T}$ cells observed histologically in other oncogene-driven cancer GEMMs $(19,20)$ and in the majority of human PDA $(21)$. We, therefore, systematically utilized the KPC mouse model as a means to reevaluate mechanisms of immune surveillance and tumor-host interactions in an oncogene-driven tumor with an immunosuppressive surrounding tumor stroma.

\section{Results}

$T$ cells do not affect the natural history of murine PDA. To determine whether $\mathrm{T}$ cells affect the natural history of PDA in the KPC model, we serially administered CD4- and CD8-depleting antibodies or an isotype control to juvenile (3- to 5-week-old) KPC mice. Treated mice were then monitored by ultrasound for the development of PDA and evaluated for signs of morbidity (Figure 1, A and B). Efficacy of antibody-mediated T cell depletion for the duration of the study was confirmed (Supplemental Figure 1; supplemental material available online with this article; doi:10.1172/jci.insight.88328DS1). Tumor-free survival (i.e., time to diagnosis) and overall survival were statistically indistinguishable between CD4/CD8-depleted mice and isotype-treated mice (Figure 1C). Likewise, no difference was observed for tumor-free and overall survival in mice exclusively depleted of CD8 T cells (Figure 1C). Median time to diagnosis was 105 days, 93 days, and 110 days for isotype-treated, CD4/CD8-depleted, and CD8-depleted mice, respectively; median overall survival was 147 days, 142 days, and 139 days for isotype-treated, CD4/CD8-depleted, and CD8-depleted mice, respectively. Tumors from each cohort exhibited similar histology, and flow cytometry demonstrated the same prominent leukocytic infiltrate in isotype control versus T cell-depleted mice (Figure 1, D and E). These results differ from classic mouse sarcomas in which immunodeficient hosts exhibit a greater frequency of tumors and decreased survival $(2,22)$; however, carcinogen-driven mouse sarcomas have a hypermutator phenotype and strong $\mathrm{T}$ cell reactivity (3).

To test further for $\mathrm{T}$ cell immunosurveillance, we created cell lines derived from the tumors of immune-competent KPC mice. Following tumor harvest and elimination of stromal elements in culture, a representative line ( 4662 cells) was verified by RT-PCR to express the recombinant mutant alleles. This low-passage cell line was then implanted subcutaneously into $\mathrm{T}$ cell-depleted or isotype-treated syngeneic mice and followed for 21-24 days to assess tumor growth (Figure 2A). Similar to our findings in the autochthonous KPC model, tumor growth of 4662 cells was unaffected by $\mathrm{T}$ cell depletion of recipient mice (Figure 2B). Tumor growth featured rapid recapitulation of the dense, stromal microenvironment of the original tumor despite elimination of nontumor cells from the cell line (Figure 2C). Similarly, the extracellular matrix of 4662 PDA tumors is extensive (23).

To exclude the possibility that the initial bolus of $5 \times 10^{5}$ tumor cells overwhelmed an otherwise relevant immune response, we also tested a lower subcutaneous dose of $10^{5} 4662$ cells. Again, tumors grew with similar kinetics in the presence or absence of T cells (Figure 2B). 4662 tumors also grow with similar kinetics in $\mathrm{NOD} / \mathrm{SCID} / \mathrm{\gamma c}^{-1-}$ immune-incompetent mice compared with wild-type mice (23). To assess whether the negative immune checkpoint molecules PD-1 and CTLA-4 were influencing tumor rejection, as observed in the MCA model (24), we also repeated these experiments in the presence of mAb blocking PD-1 and CTLA-4. In our system, this treatment alone did not enable tumor rejection (Supplemental Figure 2). Furthermore, we found that 4662 cells remain responsive to IFN- $\gamma$ by upregulating MHC class I (but not MHC class II) (Supplemental Figure 3).

To elucidate the mechanism of tumor outgrowth in the KPC model, we assessed immunoediting directly by generating cell lines from tumors arising in chronically T cell-depleted KPC mice and implanting them in immune-competent or T cell-depleted syngeneic hosts (Figure 2A and Supplemental Figure 1) - again, following an experimental design close to that used in the MCA model. Each cell line derived from a T cell-depleted KPC mouse (1262, 1493, and 1638) grew with similar kinetics regardless of the immune status of the recipient mouse, and no tumor regression was observed in any individual mouse (Figure 2D). In contrast, tumor cell lines generated from T cell-deficient mice in immunogenic models (such as MCA, LMP-1, or LoxP-Tag models) are rejected between $40 \%$ and $100 \%$ of the time $(2,4,5)$ upon implantation in wild-type hosts. Our findings, therefore, implicate an alternative mechanism of tumor outgrowth other than immunoediting or tolerance.

$K P C$-derived cell lines lack predicted neoepitopes. We hypothesized that the divergence of KPC tumors from classical "triple E" features of cancer immune surveillance reflects differences in antigenicity, which can regulate progression-free survival and $\mathrm{T}$ cell infiltration in other mouse models $(22,25,26)$. Moreover, anti- 
A

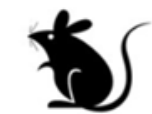

Young KPC

(3-5 weeks)

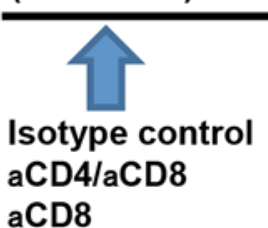

aCD8

C

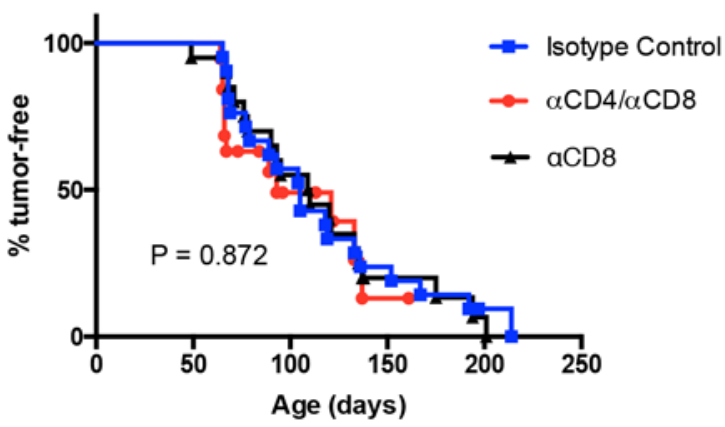

D
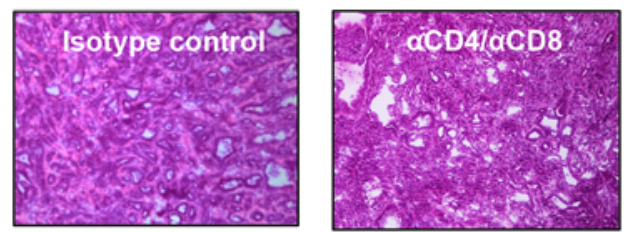

Serial ultrasound (every 2 weeks)

$T$ cell depletion (every 4 days)
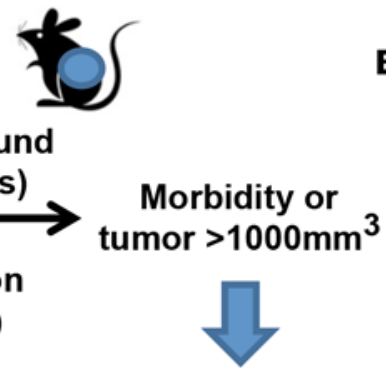

euthanasia
B

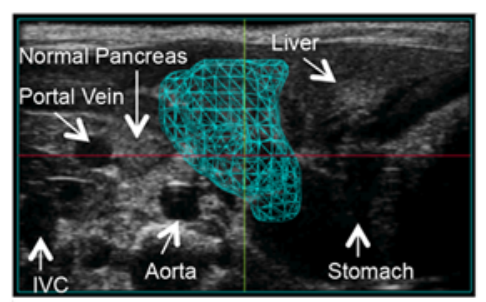

\section{Overall survival}
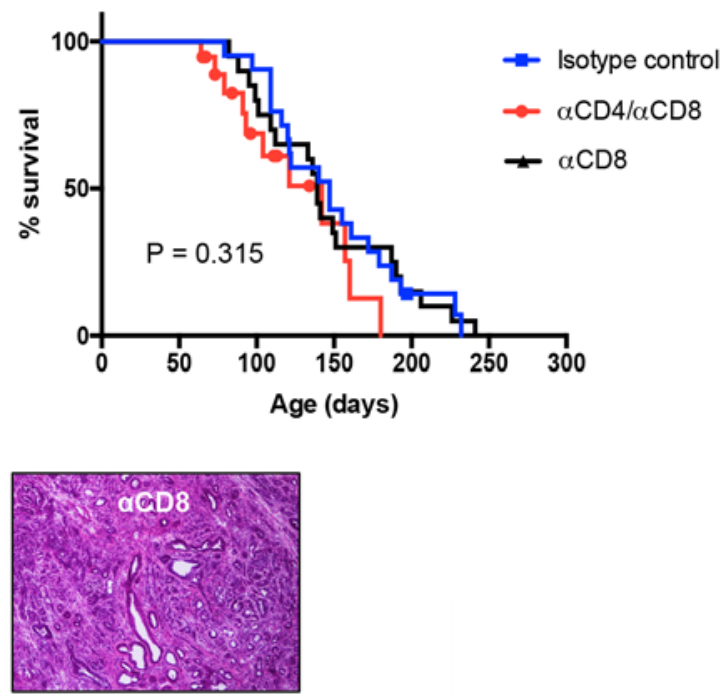

E

CD45+ Leukocytes
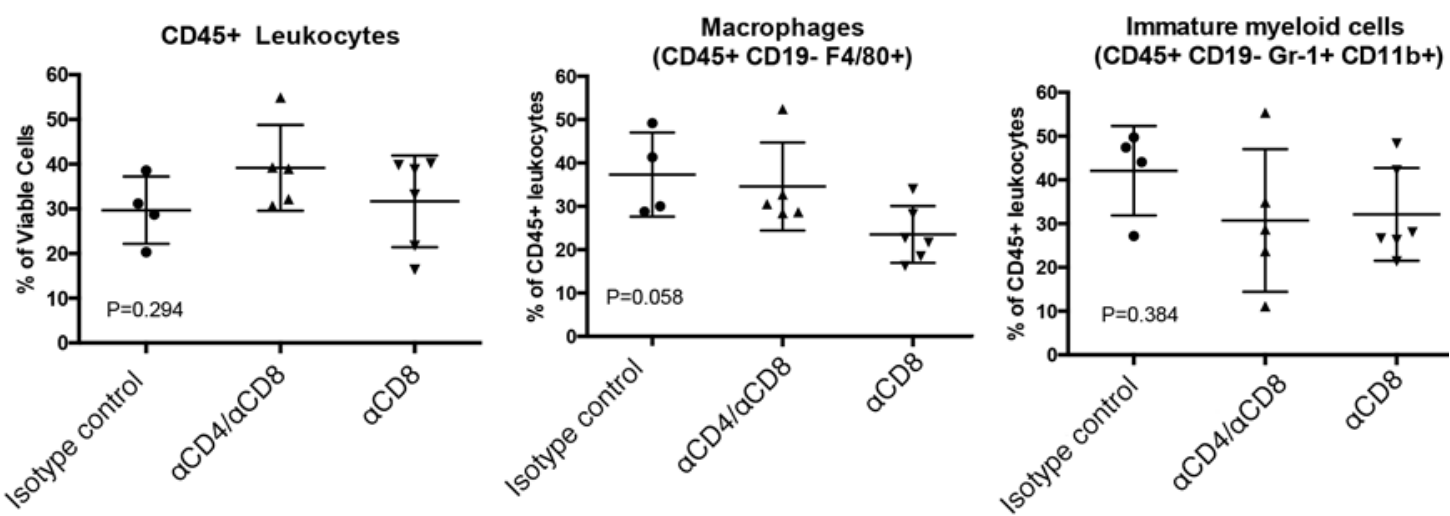

Figure 1. T cell dependency of KPC pancreatic cancer. (A) Experimental design for survival studies of syngeneic KPC mice treated with an isotype control antibody, depleted of CD4 and CD8 T cells ( $\alpha$ CD4/ $\alpha$ CD8), or depleted of CD8 T cells alone ( $\alpha$ CD8) beginning at 3-5 weeks of age. $n=19-21$ mice per cohort. Starting at 7-8 weeks of age, mice were monitored by ultrasound every other week for tumor development and examined daily for morbidity. (B) Representative image of a tumor at the time of diagnosis by abdominal ultrasound (volume $=19 \mathrm{~mm}^{3}$ ). IVC, inferior vena cava. (C) Tumor-free survival according to ultrasound monitoring (time to diagnosis) and overall survival according to daily monitoring for the 3 cohorts described in $\mathbf{A}$. $P$ values were determined by log-rank (Mantel-Cox) analysis. (D) H\&E staining of a representative pancreatic tumor from each treatment cohort (original magnification, $\times 10$ ). (E) Flow cytometric analysis of tumors at the time of euthanasia (4-6 mice per cohort) to assess infiltration by leukocytes (CD45 ${ }^{+}$cells as percentage of viable

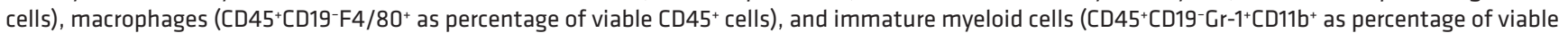
CD45 ${ }^{+}$cells). Data are shown as whisker plots (symbols represent individual experimental measurements; center line, mean; outer lines; 1 SD), with $P$ values determined by 2-way ANOVA. 


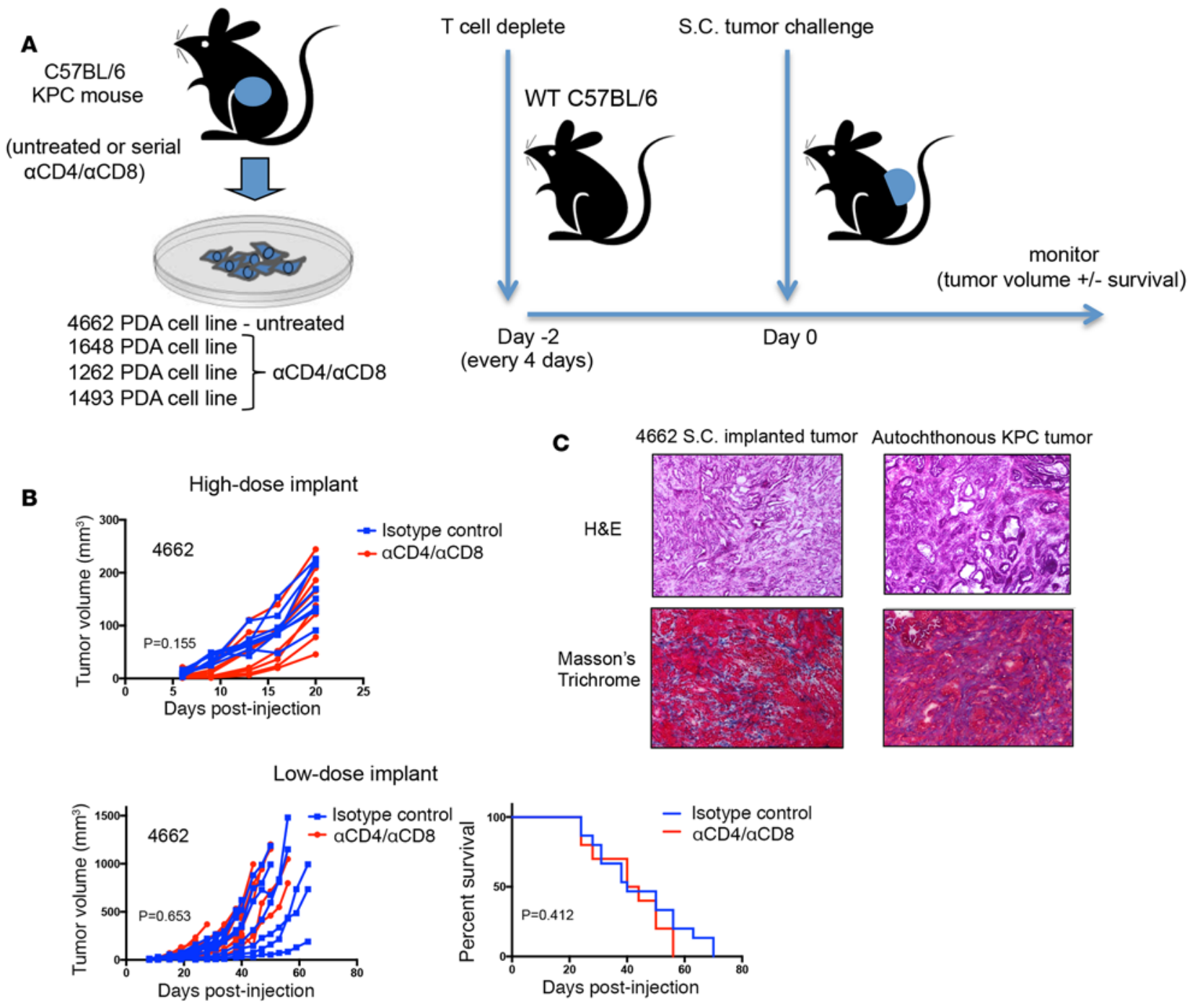

D
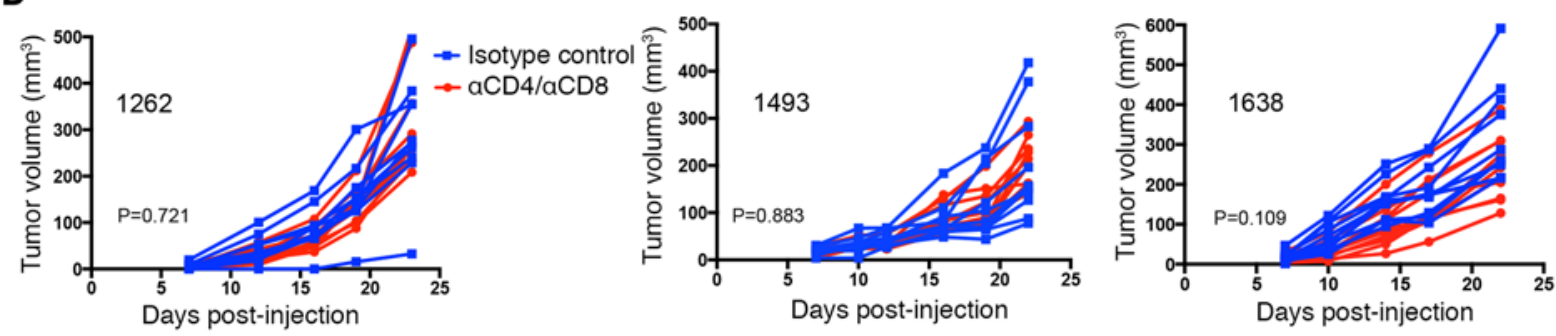

$\mathbf{E}$

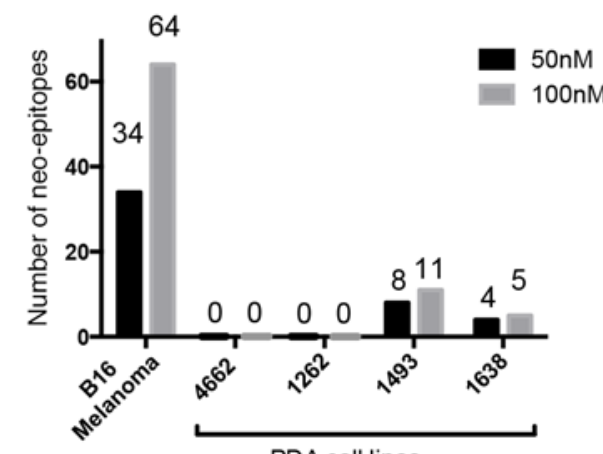

PDA cell lines 
Figure 2. KPC-derived tumor cell lines grow progressively upon implantation, regardless of donor or host T cell status. (A) Experimental design using syngeneic pancreatic ductal adenocarcinoma (PDA) cell lines generated from immune-competent KPC mice or KPC mice serially depleted of CD4/CD8 T cells beginning at 3-5 weeks of age, as shown in Figure 1A. Cell lines were implanted subcutaneously (S.C.) in syngeneic mice predepleted of CD4 and CD8 T cells or administered an isotype control antibody ( $n=8-10$ mice per cohort). Tumor growth was measured by caliper over time, and mice were monitored for overall survival. (B) Subcutaneous tumor growth of 4662 PDA cells in immune-competent syngeneic hosts (Isotype control) or immune-compromised mice $(\alpha \mathrm{CD} 4 / \alpha \mathrm{CD} 8)$, shown with high inoculum $\left(5 \times 10^{5}\right.$ tumor cells) or low inoculum ( $10^{5}$ cells). For the lower dose, mice were also monitored for overall survival. Growth data are shown as spaghetti plots of individual mice, and $P$ values indicate analysis by 2-way ANOVA. Survival data were analyzed by logrank (Mantel-Cox) test. (C) Histology of 4662 implanted tumor after 3 weeks of growth (left panels) stained by H\&E and Masson's trichrome. Right panels show H\&E and trichrome staining of an autochthonous KPC tumor. Original magnification, $\times 10$. (D) Subcutaneous growth of CD4- and CD8-depleted KPC cell lines (1262, 1493, and 1638; generated as described in A) in immune-competent syngeneic isotype control or $\alpha$ CD4/ $\alpha$ CD8 mice. $n=8-10$ mice per cohort. $P$ values shown were generated by 2-way ANOVA. (E) Numbers of predicted neoepitopes for the B16 murine melanoma cell line, the 4662 PDA cell line derived from an immune-competent KPC mouse, and 3 cell lines derived from T cell-depleted KPC mice $(1262,1493$, and 1638). Predictions are shown for both the $50 \mathrm{nM}$ binding threshold (black bars) and $100 \mathrm{nM}$ threshold (gray bars).

genicity may be related to the incidence of $\mathrm{T}$ cell neoepitopes arising from tumor missense mutations (3, 27-29), because such mutations drive strong antitumor T cell responses in the MCA model, leading to antigen loss as a means of escape consistent with immunoediting (3). We therefore performed whole-exome sequencing (WES) on 4662 PDA cells as well as the 3 PDA cell lines derived from T cell-depleted KPC mice to assess for antigenicity and immunoediting on a genomic level. As anticipated from WES analysis of tumors from other Kras-driven GEMMs (30) and human PDA (31-33), we found that 4662 PDA cells exhibited a relatively low mutational burden. Among the 20 missense mutations identified, none were predicted to comprise an 8- to 10-amino acid epitope with high affinity for MHC class I H2- $\mathrm{D}^{\mathrm{b}}$ or $\mathrm{H} 2-\mathrm{K}^{\mathrm{b}}$ molecules based on the previously defined cutoff of $50 \mathrm{nM}$ (Figure 2E) $(3,30)$. T cell-depleted PDA cell lines 1262, 1493, and 1638 also exhibited a low mutational burden, with 11, 145, and 23 missense mutations, respectively, with 0 (line 1262) or few of these mutations ( 8 for line 1493 and 4 for line 1638) predicted to generate a neoepitope (Figure 2E). Similar findings were obtained using a binding affinity cutoff of $100 \mathrm{nM}$ (Figure 2E). As a positive control in our analysis pipeline, we determined that murine B16-F10 melanoma tumors expressed 805 missense mutations, 34 of which were predicted to trigger specific $\mathrm{T}$ cell reactivity at the $50 \mathrm{nM}$ cutoff and 64 of which were predicted to trigger specific T cell reactivity at the $100 \mathrm{nM}$ threshold (Figure 2E). A prior study of B16-F10 melanoma tumors similarly found $>500$ missense mutations, and at least $30 \%$ of those experimentally tested were predicted to trigger specific T cell reactivity (34). In further contrast, MCA tumor cell lines established from immunodeficient $R a g 2^{-/-}$mice exhibited $>2,000$ somatic, missense mutations (3). Our findings, therefore, suggest that the T cell-independent growth properties of KPC-derived PDA tumors may be a consequence of the low incidence of missense mutations at baseline and subsequent scarcity of neoepitopes.

Expression of a strong antigen in a KPC tumor induces CD8-dependent tumor elimination. Based on these findings, we concluded that immune escape does not drive the natural history of PDA in this model and hypothesized that the lack of a strong neoantigen is the critical feature that defines such "immunologically cold" cancers, rather than $\mathrm{T}$ cell dysfunction or immunosuppression. We therefore predicted that introducing a strong neoantigen into the same model of PDA would prompt a $\mathrm{T}$ cell-dependent response and reproduce key findings from the MCA-driven model. To test this prediction, we retrovirally transduced 4662 PDA cells with an ovalbumin-expressing (OVA-expressing) construct (35) labeled with a Td-tomato marker to create PDA cells that express full-length OVA for processing and presentation on MHC. In particular, the OVA protein includes a well-known immunodominant MHC class I epitope (peptide SIINFEKL) that binds with high affinity to $\mathrm{H} 2-\mathrm{K}^{\mathrm{b}}$ and mimics immunological properties of a strongly immunogenic tumor neoepitope (36).

We sorted cells for expression of Td-Tomato and established tumor cell clones (rather than polyclonal tumor lines). A representative clone (V6.Ova) was selected for further studies (Figure 3, A and B, and Supplemental Figure 4). Negative sorting was used to derive a cell line ("OvaNeg") to be used as a control (Supplemental Figure 4). Expression of MHC class I and PD-L1 was similar in V6.Ova and parental 4662 cell lines and was comparably upregulated following stimulation with IFN- $\gamma$ (Supplemental Figure 3). V6.Ova cells were found to process and present SIINFEKL on H2-Kb, based on reactivity with a fluorochrome-labeled $\mathrm{mAb}$ specific for the peptide-MHC complex (Figure 3B). MHC class II was not found to be expressed on either cell line upon treatment with IFN- $\gamma$ (Supplemental Figure 3). Subcutaneous implantation of V6.Ova cells, even at a high dose ( $10^{6}$ cells), resulted in lymphocytic infiltration at days $8-9$, followed by complete tumor rejection; however, depletion of CD8 T 
A

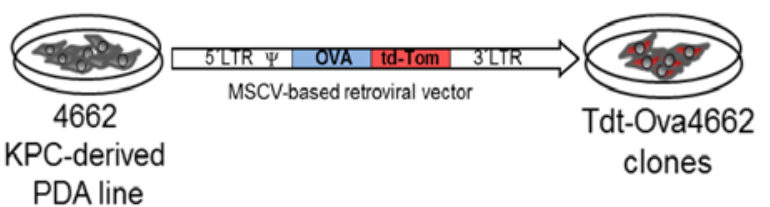

B

4662 Parental

V6.Ova clone

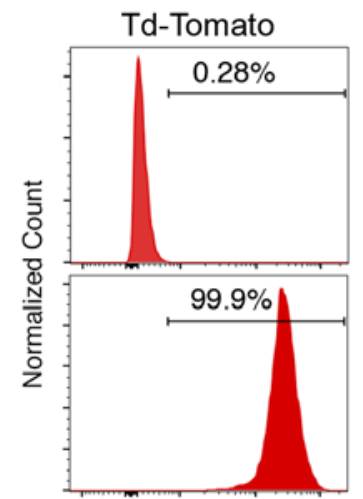

H2-Kb-SIINFEKL

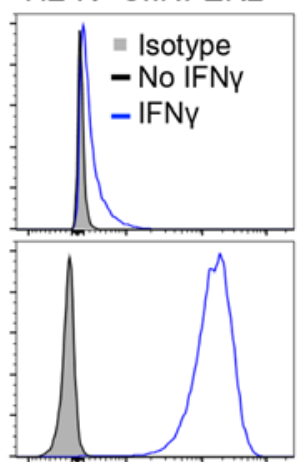

C

Subcutaneous growth of V6.Ova

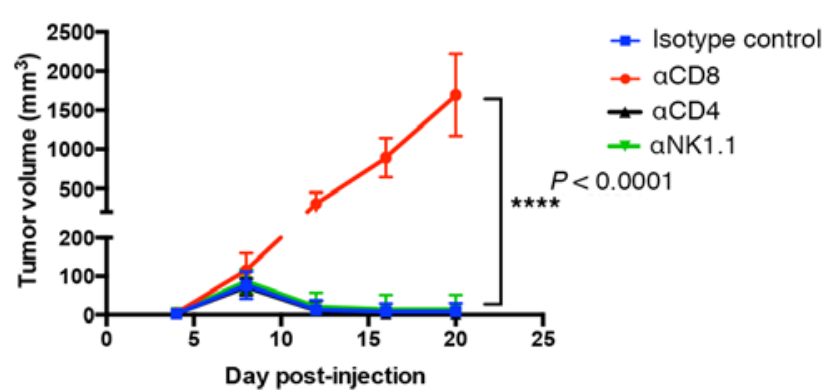

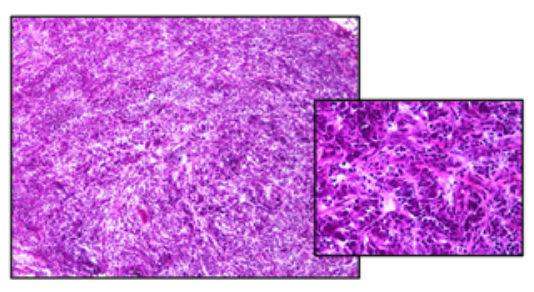

Day 8

D
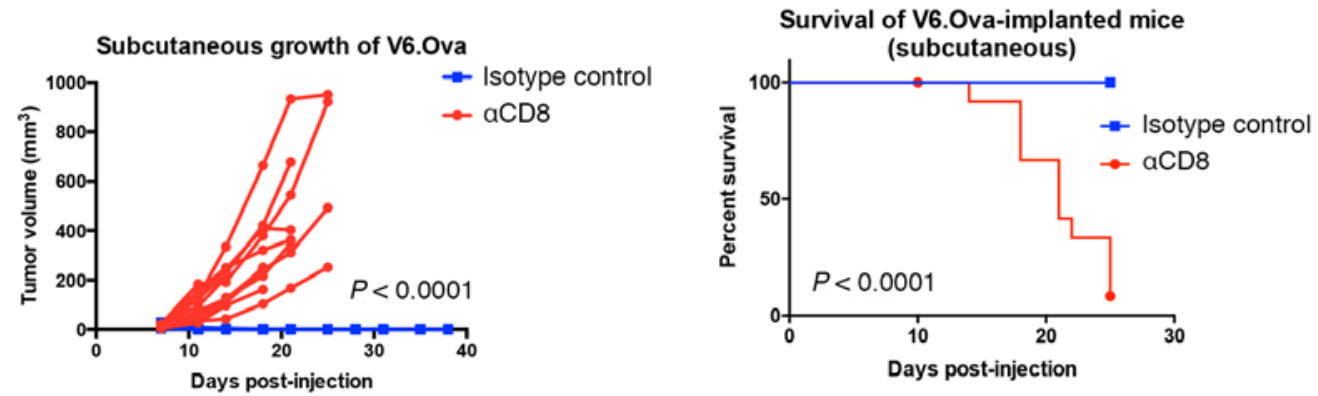

E

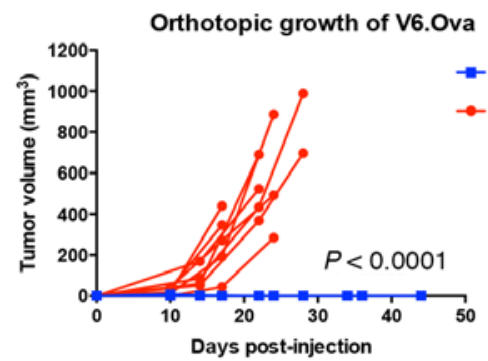

Survival of V6.Ova-implanted mice (orthotopic)
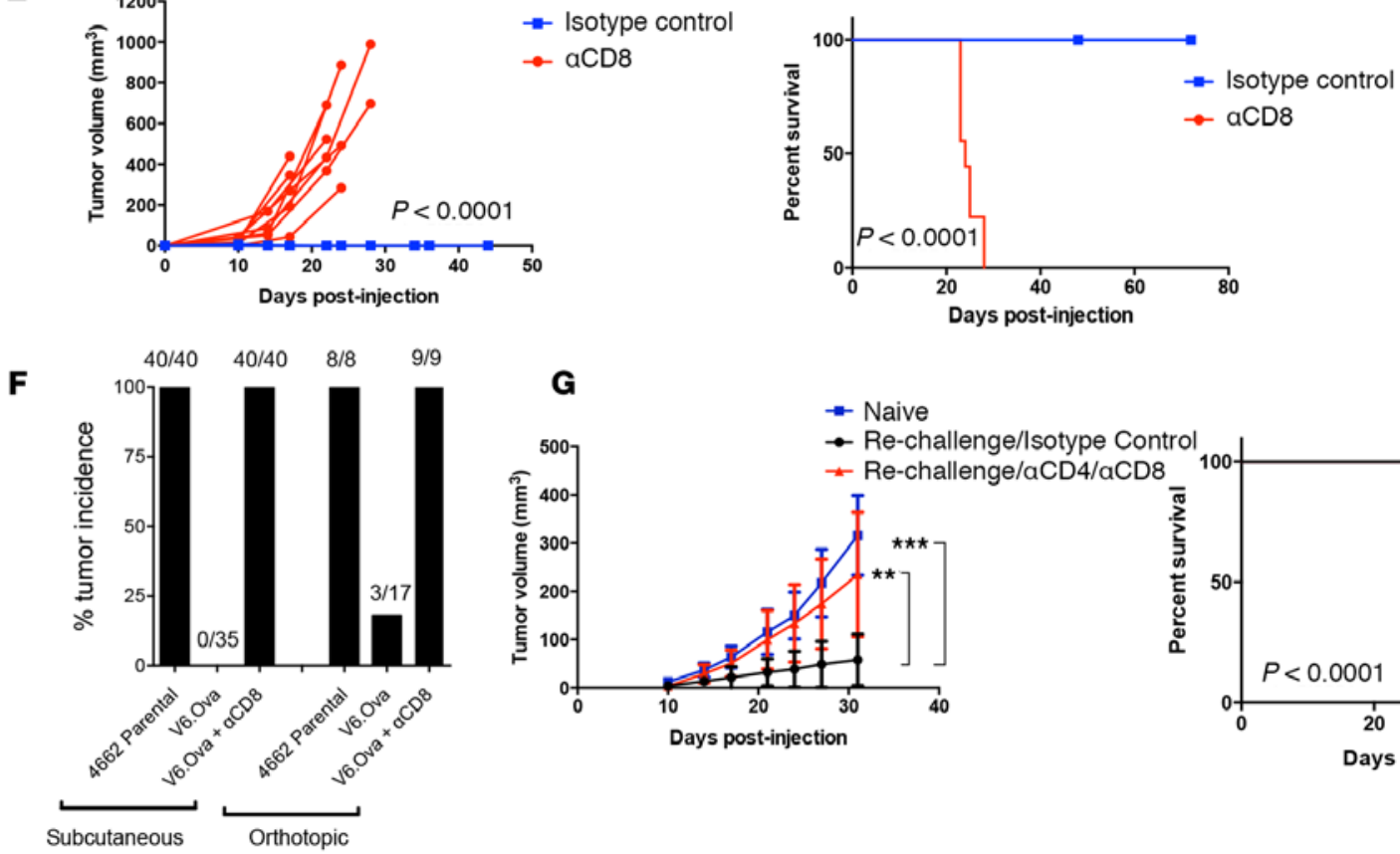

G

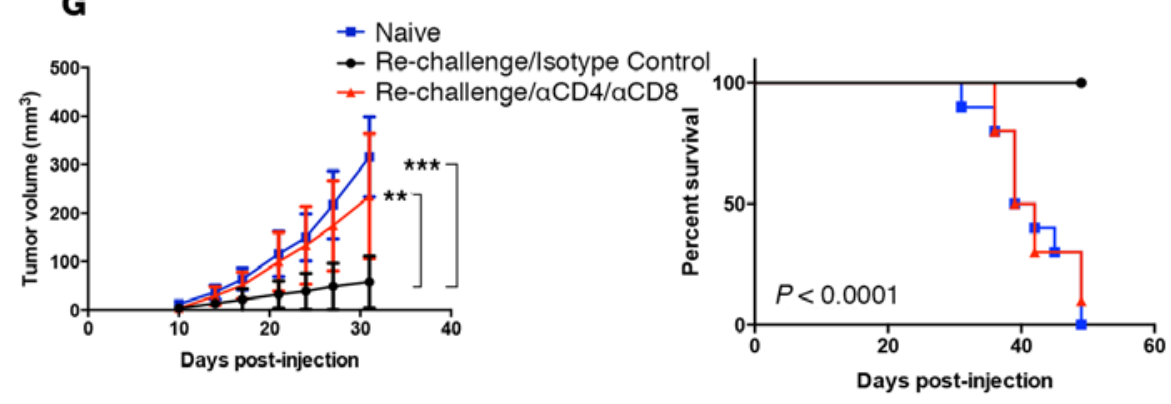


Figure 3. Expression of a strong antigen in a KPC tumor induces CD8-dependent tumor elimination. (A) Parental 4662 cells were retrovirally transduced with a Td-Tomato/ovalbumin-expressing (Tdt/OVA-expressing) construct and sorted to generate single-cell clones. (B) Flow cytometric analysis of the parental 4662 cell line compared to the V6.Ova clone. Cells were assessed for expression of Td-tomato, incubated with or without IFN- $\gamma$, and stained using a mAb against SIINFEKL-bound $\mathrm{H2}-\mathrm{K}^{\mathrm{b}}$ (MHC class I) and gated on viable (Live/Dead aqua-negative) cells. Data are representative of 3 independent experiments. (C) A high dose of the V6.0va clone ( $10^{6}$ cells) was subcutaneously implanted in syngeneic mice treated with an isotype control antibody, CD8-depleting antibody ( $\alpha$ CD8), CD4-depleting antibody ( $\alpha \mathrm{CD} 4)$, or an NK cell-depleting antibody ( $\alpha$ NK1.1) and monitored for growth over time by caliper. $n=7-8$ mice per cohort (mean tumor volume was plotted with error bars representing $\pm 1 \mathrm{SD} ;{ }^{* * *} P<0.0001$ by 2 -way ANOVA) (left). H\&E staining of an implant at day 8 of an isotype-treated mouse showing presence of tumor cells with marked infiltration of lymphocytes (original magnification, $\times 10$; inset, $\times 40$ ) (right). (D) Tumor growth at a lower inoculum of $0.75 \times 10^{6} \mathrm{~V} 6.0$ va cells was assessed in isotype-treated and CD8-depleted cohorts, which were also monitored for overall survival ( $n=12-13$ mice per cohort; $P$ value by log-rank [Mantel-Cox] test). (E) Growth of V6.Ova tumor cells implanted orthotopically in mice treated with isotype control or $\alpha \mathrm{CD} 8$ with an inoculum of $0.125 \times 10^{6}$ cells. Mice were monitored for tumor growth by ultrasound and assessed for overall survival. $n=9-10$ mice per cohort; data shown are pooled from 2 independent experiment experiments. $P$ values were determined by 2 -way ANOVA (tumor growth) and log-rank (Mantel-Cox) (overall survival). (F) A summary of subcutaneous and orthotopic growth of parental 4662 cells and isotype-treated or CD8-depleted V6.Ova-implanted cohorts. Numbers above bars indicate the number of mice growing tumors over the total number of mice tested. (C) C57BL/6 mice that rejected a subcutaneous V6.0va implant after 6 weeks were either CD4/CD8 depleted or administered an isotype control and then rechallenged with parental 4662 on the opposite flank. A third naive cohort was simultaneously challenged with parental 4662 cells at the same dose of $10^{5}$ cells. $n=9-10$ mice per cohort. Mice were followed by caliper for tumor growth and monitored for overall survival. $P$ values represent analysis by 2-way ANOVA or log-rank (Mantel-Cox) tests, respectively; ${ }^{* *} P<0.05 ;{ }^{* *} P<0.001$.

cells prior to implantation permitted rapid outgrowth of V6.Ova tumors (Figure 3C). Administration of neither $\alpha \mathrm{CD} 4$ - nor $\alpha$ NK1.1-depleting antibodies prevented tumor rejection (Figure $3 \mathrm{C}$ ), suggesting that CD8-dependent immunity was necessary and sufficient for rejection of V6.Ova cells. We used flow cytometry to quantify leukocytic infiltration for both parental 4662 and V6.OVA tumors at day 9 after implantation. Although CD45 infiltration was unchanged, we found statistically significant increases in CD3 T cells, CD8 T cells, and OVA tetramer ${ }^{+} \mathrm{CD} 8 \mathrm{~T}$ cells comparing V6.OVA cells with 4662 cells; in contrast, we found statistically significant decreases in CD4 FoxP3 cells, CD11b ${ }^{+}$cells, and $\mathrm{CD} 11 \mathrm{~b}^{+} \mathrm{Gr} 1^{+}$cells (Supplemental Figure 5). We also tested a lower dose of V6. Ova cells $\left(7.5 \times 10^{5}\right.$ cells) and likewise observed poor survival when CD8 cells were depleted, in contrast to the $100 \%$ cure and long-term survival of isotype-treated mice implanted with this number of V6.Ova cells (Figure 3D). Similar findings were observed with 2 other 4662-derived Ova-expressing clones (Supplemental Figure 4). Furthermore, we demonstrated that immunologic tolerance to OVA alone abrogated rejection of V6.Ova tumor cells, as tumors grew progressively upon transplantation into Act-mOVA mice, which possess a transgene expressing full-length OVA under the $\beta$-actin promoter (37), or into mice in which OVA tolerance was induced orally (Supplemental Figure 6).

To assess whether this antitumor response could be recapitulated in the pancreatic microenvironment, orthotopic implantation and ultrasound monitoring for tumor growth were performed in syngeneic mice. Parental 4662 cells grew rapidly in all experiments when implanted in the pancreata of wild-type mice and recapitulated the histology of autochthonous KPC tumors (Supplemental Figure 7). In contrast, 14 of 17 immune-competent mice (82\%) tested in multiple independent experiments rejected the same dose of orthotopically implanted V6. Ova cells and achieved long-term survival (Figure 3, E and F, and Supplemental Figure 7). Antibody-mediated depletion of CD8 T cells abrogated this effect, such that orthotopically implanted V6. Ova cells grew aggressively and required euthanasia of mice in all cases (Figure 3E). Thus, OVA expression by tumor cells is sufficient to mediate and maintain CD8-dependent tumor rejection in multiple microenvironments, including the pancreas.

Moreover, mice cured of V6.Ova subcutaneous implantation rejected or resisted rechallenge with 4662 parental cells, an effect lost with CD4/CD8 cell depletion immediately prior to rechallenge (Figure $3 \mathrm{G}$ ). These data are consistent with epitope spreading, whereby $\mathrm{T}$ cell immunity can be established against 4662 "self" (nonmutated) antigens in the setting of a strong, acute inflammatory immune response. In other words, the immune response to OVA cultivates the evolution over time of immunity against covert antigens liberated in an immunostimulatory setting. These data further underscore that, as previously observed in the setting of chemotherapy and CD40 agonists (16), PDA tumor cells express otherwise immunologically quiescent endogenous (non-OVA) antigens that are nevertheless capable of mediating $\mathrm{T}$ cell-dependent tumor rejection when provided with the necessary immunological impetus.

Expression of a strong antigen in PDA restores tumor immunoediting and escape. Although these findings with the V6.Ova clone recapitulated critical features of the elimination phase of immunosurveillance, the powerful antitumor response may have masked potential immune escape and thereby truncated the immunoediting process. To allow further study of the escape phase, we mixed V6. Ova cells with OvaNeg cells at high 


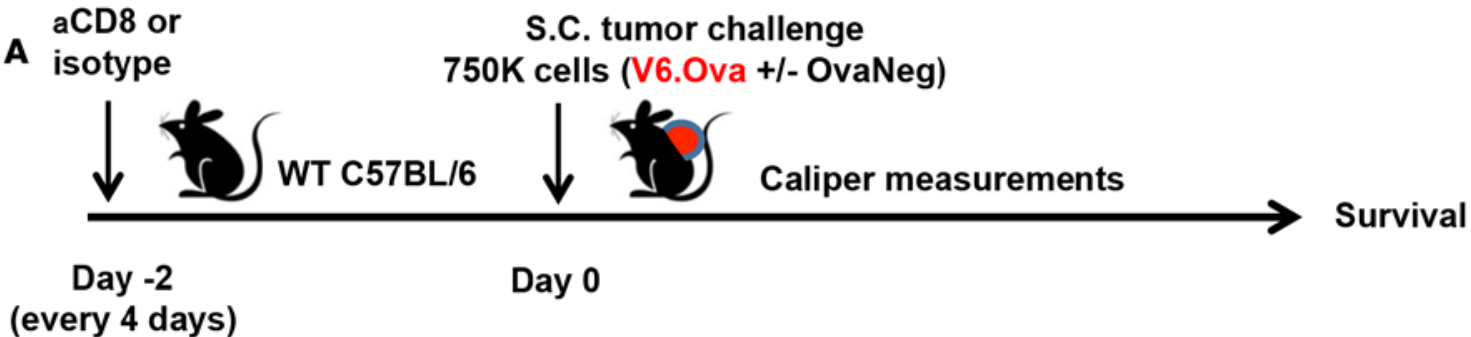

B

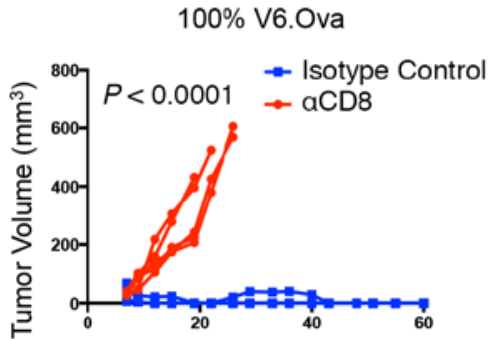

Days post-injection

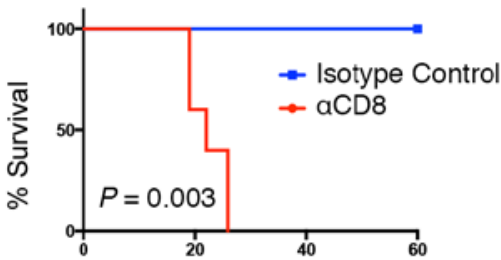

$90 \%$ V6.Ova
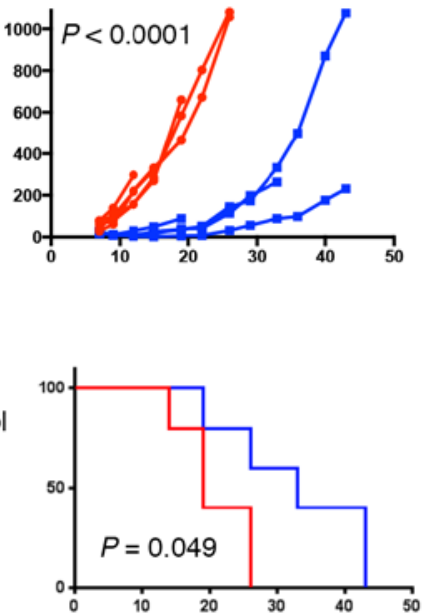

$80 \%$ V6. Ova
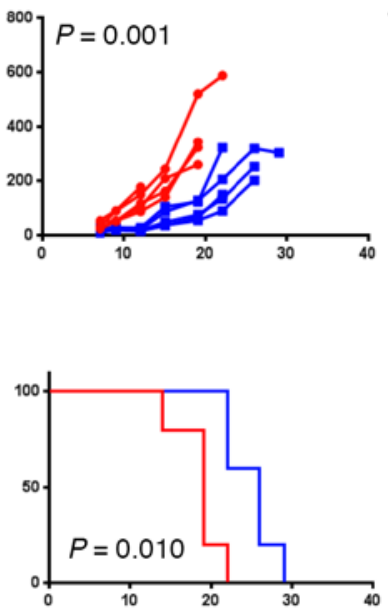

OvaNeg
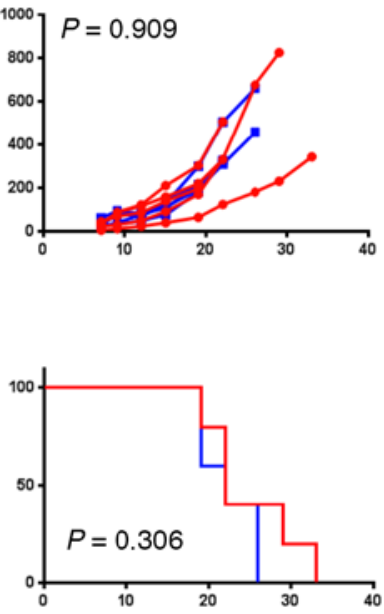

Figure 4. Selective outgrowth of Ova- tumors in immune-competent mice. (A) Experimental design of a competition assay between V6.0va cells and negatively sorted (OvaNeg) cells subcutaneously implanted at the following ratios in immune-competent or CD8-depleted cohorts ( $n=5$ mice per cohort): $100 \%$ V6.0va (7.5 $\times 10^{5}$ V6.Ova cells); 90\% V6.0va/10\%OvaNeg (6.75 × $10^{5}$ V6.0va cells and $0.75 \times 10^{5}$ OvaNeg cells); $80 \%$ V6.0va/20\%0vaNeg $\left(6.0 \times 10^{5}\right.$ V6.Ova cells and $1.5 \times 10^{5}$ OvaNeg cells); and 100\%OvaNeg (7.5 × $10^{5}$ OvaNeg cells). (B) Implants containing either a combination of Ova+ and Ova- cells (90\% V6.Ova and $80 \% \mathrm{~V} 6.0 \mathrm{va}$ ) or a $100 \%$ population of V6.Ova or OvaNeg cells were assessed for tumor growth. Data are shown as the individual growth curves for each mouse per cohort ( $n=5$ mice per cohort). Three independent experiments were performed. $P$ values represent 2-way ANOVA. Overall survival was assessed by log-rank (Mantel-Cox) for each cohort.

ratios (90\% V6.Ova/10\% OvaNeg and $80 \%$ V6.Ova/20\% OvaNeg) for a constant subcutaneous dose of $7.5 \times 10^{5}$ cells (Figure 4A). Pure populations of both the V6.Ova clone and the OvaNeg cells were included for comparison, and each cohort was treated with $\alpha \mathrm{CD} 8$ antibody or an isotype control. Despite exhibiting slower growth compared with parental 4662 or OvaNeg cells, tumors ultimately emerged from each $90 \%$ V6.Ova or $80 \%$ V6. Ova isotype implant (Figure 4B). In each case, with CD8 depletion, tumor growth accelerated to the same rate as that observed for the pure population of $\mathrm{Ova}^{-}$cells treated with or without CD8 depletion (Figure 4B). Overall survival was likewise intermediate in the $90 \%$ and $80 \%$ V6. Ova cohorts compared with either pure population of cells (Figure 4B). The only cohort to achieve cure and long-term survival was the CD8-replete cohort implanted with $100 \%$ V6.Ova, as anticipated.

To understand antigen-specific responses in these experiments, we established tumors after administration of $80 \%$ V6. Ova cells and control lines over the course of 2 weeks, at which point complete remission was achieved in mice administered 100\% V6.Ova cells. Peptide-MHC tetramer staining for the presence of intratumoral Ova-specific $\mathrm{CD} 8^{+} \mathrm{T}$ cells revealed a 40 -fold enrichment of tetramer ${ }^{+}$cells in the tumors from $80 \%$ V6.Ova implants compared with the spleens from the same mice, tumors from mice implanted with an equivalent dose of parental 4662 cells, or tumors from mice depleted of CD8 T cells (Figure 5A), thereby demonstrating a robust adaptive immune response in vivo. Tumor-infiltrating Ova-specific $\mathrm{CD} 8^{+}$ $\mathrm{T}$ cells prominently expressed granzyme $\mathrm{B}$ and Tbet, consistent with an effector phenotype (Figure 5B). Moreover, these Ova-specific CD8 T cells were highly functional, even after 3 weeks, expressing Ki67 and IFN- $\gamma$ upon stimulation ex vivo (Figure 5B). Overall, this effector phenotype was most prominent in the tumor compared with the spleen (Figure 5B). These results suggest a highly specific CD8 T cell response to Ova-expressing cells that bypasses $\mathrm{Ova}^{-}$cells. 

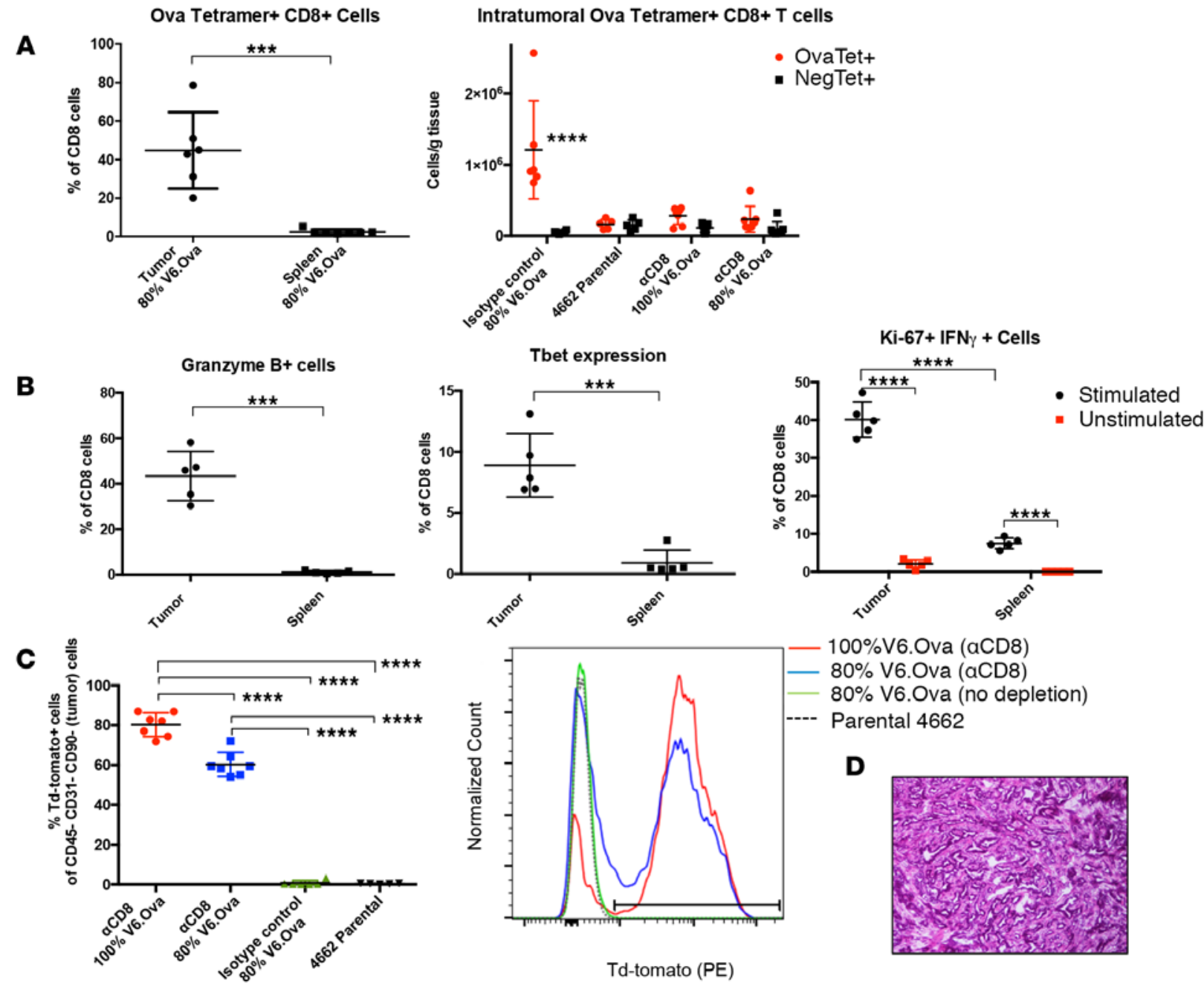

OvaTet+

- NegTet+

Figure 5. Immunity is dependent on cytotoxic Ova-specific CD8 T cells. (A) From the experimental schema in Figure 4A, Ova-specific CD8 ${ }^{+} \mathrm{T}$ cells in $80 \%$ V6.OVA tumors at day 14 were compared with spleens and quantified in the tumor in all cohorts. $n=5-7$ mice per cohort; data are representative of 3 independent experiments. ${ }^{* *} P<0.001$ by unpaired 2-tailed Student's $t$ test. ${ }^{* * *} P<0.0001$ by 2 -way ANOVA. Data are shown as whisker plots (symbols represent individual experimental measurements; center line, mean; outer lines; $1 \mathrm{SD}$ ). (B) Intratumoral CD8 ${ }^{+} \mathrm{T}$ cells in $80 \%$ V6.0va-implanted mice were assessed by flow cytometry for intracellular levels of granzyme B and Thet at day 21 . Ki67 and IFN- $\gamma$ expression was also assessed with or without stimulation with PMA/ionomycin. $n=6$ mice per cohort. Data are representative of 2 independent experiments. ${ }^{* *} P<0.001$ by unpaired 2-tailed Student's $t$ test ${ }^{* * * *} P<0.0001$ by 2 -way ANOVA. (C) Tumor-enriched live cells (CD45-CD31-CD90-) were assessed for Td-Tomato expression by flow cytometry; Td-tomato ${ }^{+}$ cells are shown as a percentage of this tumor-enriched population across cohorts. $n=5-7$ mice per cohort. Data are representative of 3 independent experiments. ${ }^{* * *} P<0.0001$ calculated by 2-way ANOVA. Representative data are shown as a histogram of Td-tomato expression for each cohort. (D) H\&E stain of an isotype-treated $80 \%$ V6. Ova tumor (original magnification, $\times 10$ ).

To determine whether the emerging tumor cells themselves had been sculpted by a host $\mathrm{T}$ cell response, we also tracked Td-tomato expression in tumors established for 2 weeks. Consistent with immunoediting, tumor cells that eventually grew from $80 \%$ V6. Ova implants in isotype-treated mice did not express Td-tomato, resulting in expression identical to that of parental 4662 tumors (Figure 5C). Moreover, the stromal architecture of these tumors resembled that of 4662 parental tumors (Figure 5D). A polyclonal 4662 cell line transfected with Td-tomato/OVA but not cloned also grew out, but progressive tumor cells did not express Td-tomato, emphasizing the experimental importance of tumor cell cloning in order to distinguish tumor outgrowth of rare antigen ${ }^{-}$tumor cells among polyclonal populations of tumor cells engineered to express antigen. Our results are consistent with the escape and subsequent proliferation of $\mathrm{Ova}^{-}$tumor cells from the original mixed implant; the data also demonstrate that elimination of antigen-rich cells can occur, even in the presence of the immunosuppressive microenvironment orchestrated by escaping tumor cells. In contrast, CD8-depleted cohorts challenged with 100\% V6.Ova and 80\% V6.Ova significantly and propor- 
tionately retained their tumor expression levels of Td-tomato (Figure 5C). Loss of Td-tomato expression in the presence of activated intratumoral $\mathrm{CD} 8^{+} \mathrm{T}$ cells likely represents selective outgrowth of the Ova ${ }^{-}$tumor cell subset that escapes $\mathrm{T}$ cell-mediated elimination.

Finally, we evaluated whether there were any potential intrinsic differences between OVA-expressing tumors and control tumor cells. Using either flow cytometry or RNA sequencing, we examined the following markers: PD-L1, IDO, cancer stemness (nanog, Sox2, Pou5F1), proliferation (Ki-67), survival associated (Bc12 and Bcl-XL), $\beta$-catenin, and Ezh2. There were no statistical differences between 4662 and 4662.OVA cells, with the exception of $\beta$-catenin, which was statistically significantly more highly expressed in 4662 .OVA cells compared with 4662 cells, but the expression was very prominent in both (Supplemental Figures 3 and 8).

\section{Discussion}

In this study, we aimed to understand the biological principles underlying immune surveillance in tumors such as pancreatic adenocarcinoma that exhibit far fewer missense mutations and potential neoepitopes than classically described carcinogen-induced, highly mutated tumors. In contrast to these immunogenic tumor models from which the "triple E" theory of elimination-equilibrium-escape was surmised (2), we found no evidence for immunoediting in a mutant Kras-driven murine model of PDA. Here, we show that KPC tumors progress the same with or without a functional adaptive immune system, similar to other mouse tumors that have also been shown to grow independently of T cells (6-9). We further show for the first time to our knowledge that KPC pancreatic tumors have few if any predicted neoepitopes derived from somatic tumor missense mutations and that an immunoediting phenotype can be rescued by ectopic expression of a neoantigen (OVA) in the same tumor model. Thus, the manifestations of cancer immune surveillance in the KPC model depend on tumor antigenic strength; the lack of neoepitopes is not a consequence of immunoediting in this model. Indeed, our data suggest that there is no selection for less immunogenicity in this model and thus no need for immunoediting; as such, this tumor model is not representative of a situation (seen in highly mutated tumors) after selection pressure has been applied to cancer cells to evade the adaptive immune response. These findings suggest an alternative biological mechanism in which tumor outgrowth reflects immune quiescence, which is linked to, and regulated by, poor tumor antigenicity - a conclusion with potential relevance to human cancers with a low burden of mutations and minimal neoepitopes.

Our experimental approach was to revisit the classic immunological experiments performed in the MCA model but using the KPC model. Thus, we examined tumor growth and development in KPC mice in the presence or absence of T cells and found no phenotype - in sharp contrast to the MCA model or other models in which T cells markedly influence tumor development (2). We then isolated KPC tumors from $\mathrm{T}$ cell-deficient mice and transplanted these into syngeneic immune-competent mice and failed to observe tumor rejection - again in contrast to the MCA model in which tumors arising in T cell-deficient mice are efficiently rejected in immune-competent mice (2). Finally, KPC tumors that grew in immune-competent mice were refractory to therapeutic checkpoint blockade, yet MCA progressor tumors can be rejected with checkpoint blockade (24).

To explain these differences between the KPC and MCA models, we found that KPC tumors express few if any neoepitopes, compared with the hundreds of such epitopes in MCA tumors, raising the hypothesis that the main regulator of immune quiescence (KPC) versus surveillance (MCA) in cancer was based on tumor antigenicity. Thus, we used the classical $\mathrm{T}$ cell antigen OVA to establish OVA-expressing KPC tumor clones and observed a full reversal of immune quiescence and reproduction of the cardinal features of the "triple E" hypothesis, including robust elimination of $\mathrm{OVA}^{+} \mathrm{KPC}$ tumor clones, despite microenvironmental immune suppression. Jacks and colleagues have also noted the importance of antigen in cancer immunosurveillance, but in their sarcoma model, even tumors without ectopically expressed antigen grew differentially in the presence or absence of $\mathrm{T}$ cells (22). In contrast to our data with KPC, sarcomas in the Jacks study develop faster and kill the mice quicker in the absence of T cells, even without the expression of an artificial antigen. In our KPC system, we started with a tumor that does not exhibit the native immunogenicity of these sarcoma models and is fully unaffected by $\mathrm{T}$ cells even at baseline. A limitation of our study is the subcutaneous or orthotopic implantation of a bolus of antigen-expressing tumor cells, which likely does not reproduce the evolution of neoepitope expression of tumor cells emerging in an immune-suppressive microenvironment. The generation of a KPC model in which OVA or another defined antigen could be introduced at various times during the natural history of the in vivo developing tumor 
will be important to clarify this point. Nevertheless, we found that the critical characteristic of OVA in the KPC system was its "foreign" or "neo" quality, because OVA-tolerant mice in 2 model systems could not reject the same OVA ${ }^{+}$KPC tumors cells, even if implanted with a bolus. Importantly, using mixtures of OVA-expressing and OVA ${ }^{-}$tumor cells, we observed that only OVA- cells escaped immune surveillance and grew - an outcome consistent with immunoediting. Thus, the tumor cell-intrinsic antigenic landscape of KPC tumors principally drives the manifestation of cancer immunosurveillance.

Intriguingly, mice that rejected OVA-expressing 4662 tumor cells were subsequently able to reject parental (non-OVA) 4662 in a CD8 T cell-dependent manner. Rejection of 4662 required time to generate $\mathrm{T}$ cell memory because 4662 parental cells were not rejected when injected at the same time with V6.OVA cells. We hypothesize that necrosis induced by cytolytic Ova-specific T cells may have released endogenous antigens in the initial immunostimulatory setting, permitting subsequent tumor rejection upon rechallenge based on epitope spreading. Importantly, resolution of the inciting antigen was critical in developing memory rather than inducing $\mathrm{T}$ cell exhaustion, the latter of which may have occurred in the mixed implant studies in which self-antigens persisted due to the escape of non-Ova cells (38). Nevertheless, these data underscore that robust immunity to endogenous antigens can under certain circumstances be fostered. Indeed, despite the lack of missense mutations in KPC-derived tumors, we previously reported that immune-modulation or disruption of the microenvironment can lead to $\mathrm{T}$ cell dependent tumor rejection of KPC tumors. A cocktail of checkpoint inhibitors ( $\alpha$ CTLA- 4 and $\alpha$ PD-1, which alone are ineffective) and an immune-stimulatory vaccine (agonistic CD40 mAb with chemotherapy) results in a high rate of cures of established KPC (non-OVA) tumors and the establishment of CD8-dependent memory responses that protected against rechallenge (16). CD40/chemotherapy and PD-1 doubles survival of mice with established spontaneous KPC tumors as well (16). T cell-mediated rejection of implanted or spontaneous KPC tumors has additionally been observed with therapeutic attenuation of multiple other immune suppressive pathways in the tumor microenvironment, including CXCL12, GM-CSF, and BTK (17, 39-41).

The clear potential of T cells to reject KPC tumors, as a model of tumors without neoepitopes, is clinically significant for two reasons. First, in light of a major focus in the field on neoepitopes as targets for cancer immunotherapeutics, these findings justify a reconsideration of self-antigens - as well as "abnormal self-antigens" not derived on the basis of nonsynonymous mutations $(42,43)$ - as potential tumor-rejection antigens. Second, without Darwinian-like pressure from $\mathrm{T}$ cells driving immune escape, the underlying pancreatic tumor cells remain highly susceptible to $\mathrm{T}$ cells, if such $\mathrm{T}$ cells can be induced. We do not mean to suggest antigen gene therapy as the translational step supported by our findings; rather, combinations of vaccines with inhibition of tumor and host immune suppression are the high priority strategies in PDA (44). In the KPC model, for example, the combination of chemotherapy and agonist CD40 mAb synergizes to generate durable remissions and $\mathrm{T}$ cell memory in the absence of neoepitopes (45), data supporting a new clinical trial in patients with metastatic pancreatic cancer (NCT02588443).

In summary, we demonstrate that the biological consequences of immune-tumor cell interactions largely depend on the expression of a tumor antigen of adequate strength. In KPC mice, which exhibit few if any neoantigens, $\mathrm{T}$ cells do not affect the natural history of disease, but the expression of a single, strong antigen in the tumor restores the classical "triple E" phenotype. Our findings carry important clinical implications for the design of future cancer immunotherapies, particularly for treatment-refractory tumors that are "immunologically cold."

\section{Methods}

Animals. Survival data and primary cell lines were generated from $\underline{\operatorname{Kras}}{ }^{L S L-G 12 D /+}$, Trp $253^{L S L-R 172 H /+}$, Pdx 1 - $\underline{\text { Cre }}(\mathrm{KPC})$ mice (14) bred in-house, backcrossed more than 10 generations with C57BL/6 mice (Jackson Laboratories), and assessed at the DartMouse Speed Congenic Core Facility at the Geisel School of Medicine at Dartmouth College. The raw SNP data were analyzed using DartMouse's SNaP-Map and Map-Synth software, allowing the determination for each mouse of the genetic background at each SNP location. Backcrossed KPC mice were found to be congenic based on the DartMouse Illumina GoldenGate Genotyping Assay, which interrogated 1,449 SNPs spread throughout the genome. C57BL/6 OVA transgenic mice (Act-mOVA) (38) were purchased from Jackson Laboratories. Tumor implant studies were performed using 8- to 10-week-old female C57BL/6 mice purchased from Jackson Laboratories. For induction of oral tolerance to OVA, wild-type mice were treated by oral gavage with $50 \mathrm{mg}$ OVA protein (Sigma-Aldrich) dissolved in $250 \mu \mathrm{PBS}$ or with PBS alone weekly for 3 doses, followed 1 week later by tumor challenge with $1.25 \times 10^{5}$ V6.Ova tumor cells subcutaneously. 
Collection of tissue samples from mice. Whole pancreata of KPC mice or subcutaneous tumors were dissected and processed as previously described (39). Tissues were extensively minced with a razor and incubated in collagenase IV solution ( $1 \mathrm{mg} / \mathrm{ml}$ in RPMI media) for 45 minutes at $37^{\circ} \mathrm{C}$. The dissociation reaction was stopped by 1:1 dilution with cold FCS, followed by passing cells through a $70-\mu \mathrm{m}$ cell strainer. The cell suspension was carefully passed through a 27-gauge needle, washed twice in DMEM, and passed through a second strainer prior to use for flow cytometry or cell culture.

Spleens were homogenized through a $70-\mu \mathrm{m}$ strainer and carefully passed through a 27-gauge needle to create a single-cell suspension. Following centrifugation, pelleted cells were incubated with ACK lysis buffer (BioWhittaker) at a 1:10 ratio for 8 minutes to induce red blood cell lysis. Cells were washed twice in RPMI prior to use for flow cytometry.

Peripheral blood was collected by tail vein into heparinized microhematocrit capillary tubes. Blood was removed from the tubes by centrifugation and similarly prepared for flow cytometry with ACK lysis buffer and serial washes in RPMI media.

Preparation of cell lines. Tumors from the pancreata of KPC mice were excised with sterile scissors and extensively minced prior to dissociation in $1 \mathrm{mg} / \mathrm{ml}$ collagenase IV solution at $37^{\circ} \mathrm{C}$ for 45 minutes. Dissociated cells were passed through a $70-\mu \mathrm{m}$ strainer and plated in serum-free DMEM at varying concentrations in a 6-well plate. Cells were maintained in serum-free media for at least 2 weeks and passaged to higher volume flasks for expansion in DMEM + 10\% FCS. Cells were used for implant studies at a low number (just 3-5) passages. Cell lines were validated by RT-PCR to assess for Cre-mediated recombination of the mutant Kras and Trp53 alleles. Primer sequences to detect recombined Kras and Trp53 loci are as follows: forward 5'-gtctttccccagcacagtgc-3' and reverse 5'-ctcttgcctacgccaccagctc-3', and forward 5'-agcctgcctagcttcctcagg- $3^{\prime}$ and reverse 5 '-cttggagacatagccacactg-3', respectively.

In vivo mouse studies. T cell depletion of either KPC or C57BL/ 6 mice was achieved by intraperitoneal injection of $0.2 \mathrm{mg}$ of $\alpha \mathrm{CD} 8$ (2.43), $\alpha \mathrm{CD} 4$ (Gk1.5), or an IgG2b isotype control (LTF-2) diluted in $100 \mu 1$ sterile PBS. Antibodies were purchased from BioXcell. For long-term depletion in KPC mice, pups were administered a first dose at 3-5 weeks of age and every 4 days thereafter until euthanasia. The initial depletion was verified by flow cytometry of peripheral blood and then reassessed every 2 or 3 weeks thereafter for the duration of the study; blood was drawn prior to readministration of the antibody.

For subcutaneous implant studies, mice were pretreated with $\mathrm{T}$ cell-depleting antibodies 2 days prior to tumor challenge and then every 4 days thereafter. T cell depletion was confirmed by peripheral blood samples and end-of-study flow cytometry. NK cell depletion was achieved by intraperitoneal injection of $0.2 \mathrm{mg}$ PK136 $\mathrm{mAb}$ (BioXcell) on days $-1,0$, and +1 and every 5 days thereafter. NK cell depletion was confirmed by end-ofstudy flow cytometry on splenocytes. For checkpoint blockade studies, mice were treated intraperitoneally with aPD-1 (RMP1-14, BioXcell; $0.2 \mathrm{mg}$ per dose) on days 0, 3, 6, 9, 12, 15, 18, and 21 and $\alpha$ CTLA-4 (9H10, BioXcell; $0.2 \mathrm{mg}$ per dose) on days 0,3 , and 6 after enrollment. All antibodies were endotoxin free.

KPC mice were enrolled in survival studies on a rolling basis, as described previously (18). Genotyped mice were allocated to treatment groups in a randomized fashion and monitored for development of tumor-associated morbidities, including ascites, lethargy, depression, or a tumor volume $>1,000 \mathrm{~mm}^{3}$. Mice were censored for unrelated morbidities, including prolapsed rectum or penis, non-PDA tumors (thoracic and submandibular), and malocclusion.

Diagnosis of PDA in KPC mice was performed by abdominal ultrasound beginning at 7-8 weeks of age and every other week thereafter; between scheduled ultrasounds, mice were additionally monitored by palpation and imaged when indicated. Ultrasound was performed with a Vevo 2100 imaging system with a 55-MHz MicroScan transducer (Visual Sonics). Tumors were visualized and reconstructed using the integrated Vevo Workstation software package to assess tumor volume. Mice were censored from the diagnostic study if euthanized for non-PDA morbidity prior to a definitive diagnosis.

Subcutaneous implant studies were performed using PDA cell lines titrated to grow with similar kinetics over 21-24 days at a relatively low dose $\left(<1 \times 10^{6}\right.$ cells $)$ to avoid ulceration and permit any relevant immune response ( $4662: 5 \times 10^{5}$ cells; 1638: $7.5 \times 10^{5}$ cells; $1262: 2 \times 10^{5}$ cells; $1493: 3 \times 10^{5}$ cells). Cells were harvested at 80\%-90\% confluence, washed twice in sterile DMEM, and administered subcutaneously into the right flank in $100 \mu 1$ sterile DMEM. Caliper measurements of the longest tumor dimension (length $[L]$ ) and the perpendicular dimension (width $[w]$ ) were obtained every 3 to 4 days. Tumor volume was calculated as $\left(L \times w^{2}\right) / 2$. For checkpoint blockade studies, tumors were implanted as described above and allowed to establish for 9-12 days. Mice were allocated to treatment groups such that baseline tumor volume $\left(30-100 \mathrm{~mm}^{3}\right)$ was balanced across cohorts. 
For orthotopic implant studies, $1.25 \times 10^{5}$ tumor cells were injected into the tail of the pancreas in 25 $\mu 1$ DMEM using a 0.3-cc 29-gauge insulin syringe, with visualization of a small fluid bubble to indicate successful injection without leakage. Mice were anesthetized with isoflurane, laparotomies were performed using sterile surgical technique, and the spleen and pancreatic tissue were externalized prior to injection. The peritoneum was closed using absorbable Polymend MT 5/0 suture, and the overlying skin was closed with wound clips. Tumor size was quantitated over time by serial 3-dimensional ultrasonography.

Antibodies. The following mAbs were used for flow cytometry: from BD Biosciences, mIgG2a, $\kappa$ iso-

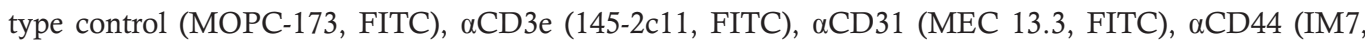
FITC), $\alpha$ CD45 (30-F11, FITC), $\alpha$ CD45 (30-F11, PE), $\alpha$ CD3 (145-2C11, PerCP), $\alpha$ CD45 (30-F11, PercP), aCD8a (53-6.7, PE-Cy7), $\alpha$ CD45 (30-F11, PE-Cy7), $\alpha$ CD45 (30-F11, APC), $\alpha C D 11 b$ (M1/70, APC-Cy7), aCD45 (30-F11, APC-Cy7), $\alpha$ CD11c, (HL3, V450), $\alpha$ CD4 (RM4-5, V450), and Gr1 (RB6-8C5, APC-Cy7); from BD Horizon, Streptavidin-V450; from eBiosciences, $\alpha \mathrm{CD} 8 \mathrm{a}$ (53-6.7, PE) and F4/80 (BM8, FITC); and from Biolegend, $\alpha \mathrm{H} 2-\mathrm{D}^{\mathrm{b}}$-Biotin (KH95), $\alpha \mathrm{Ki}-67$ (16A8, FITC), $\alpha \mathrm{H} 2-\mathrm{K}^{\mathrm{b}}$ (AF6-88.5, FITC), $\alpha \mathrm{OVA}-$ H2-K ${ }^{\mathrm{b}}$ (25-D1.16, APC), $\alpha$ CD90.2 (53-2.1, PerCP), $\alpha$ IFN- $\gamma$ (XMG1.2, PE-Cy7), $\alpha \mathrm{I}-\mathrm{A} / \mathrm{I}-\mathrm{E}$ (M5/114.15.2, PE-Cy7), $\alpha$ PD-L1 (10F.9G2, APC, BV421), $\alpha$ Granzyme B (GB11, AlexaFluor 647), $\alpha$ Tbet (4B10, Brilliant Violet 421), NK1.1 (PK136, APC-Cy7), and FoxP3 (FJK-16s, APC). H2-K ${ }^{\mathrm{b}}$ Ova Tetramer (SIINFEKL$\mathrm{PE})$ and the MHC class I-negative tetramer (APC) were purchased from MBL International Corporation (Beckman Coulter). Viability of cells was determined by staining with either 7-aminoactinomycin D (BD Biosciences) or the Live/Dead Fixable Aqua Dead Cell Stain Kit (Life Technologies).

Neoepitope prediction pipeline. Genomic DNA from KPC mouse tumor cell lines or mechanically digested control tissue (KPC spleen) was extracted using the PureLink Genomic DNA minikit (Invitrogen) and assessed for purity and yield using a NanoDrop 2000c spectrophotometer. DNA integrity was further assessed by electrophoresis on 1\% agarose gels, and DNA concentration was determined using a Qubit dsDNA BR Assay Kit (ThermoFisher Scientific). Exome enrichment was performed using an Illumina Paired End Sample Prep Kit, and library sequencing was performed using a 100-bp paired-end protocol on the Illumina platform (HiSeq2500) (High-Throughput Sequencing Center, Beijing Genomics Institute at Children's Hospital of Philadelphia). Sequence alignment and processing were performed as previously described (30). Single nucleotide variants in tumor samples were identified using MuTect (version 1.1.7) (46) with default filters against normal splenocytes from KPC mice (for PDA lines) or C57BL/6 splenocytes (for B16 melanoma). Variants were annotated using SnpEff (version $4.1 \mathrm{~L}$ ) with default settings and filtered against known SNPs. 8- to 14-amino acid sequences surrounding surviving missense mutations were then ranked for binding affinity to $\mathrm{MHC}$ class $\mathrm{I} H-2 \mathrm{D}^{\mathrm{b}}$ and $\mathrm{H}-2 \mathrm{~K}^{\mathrm{b}}$ molecules using the consensus method provided by the Immune Epitope Database and Analysis Resource (IEDB) (http://www.iedb. org/). Using 2 thresholds of potential binding affinity, peptides with a median half-maximum inhibitory concentration $\left(\mathrm{IC}_{50}\right.$ ) of less than $50 \mathrm{nM}$ or less than $100 \mathrm{nM}$ were identified as potential neoepitopes. The $\mathrm{IC}_{50}$ for each peptide was considered the median value of individual prediction method results provided by the IEDB consensus method.

$R N A$ sequencing differential gene expression analysis. RNA was isolated from tumor cell lines in biological triplicate using the Qiagen RNeasy Plus Mini Kit per the manufacturer's instructions. RNA purity and integrity were verified on an Agilent 2100 Bioanalyzer before being submitted to the Children's Hospital of Philadelphia BGI High-Throughput Sequencing Center for library construction followed by paired-end 100-bp sequencing on an Illumina HiSeq 4000 high-throughput sequencer. Alignment was performed using the STAR aligner under default settings. Raw counts of gene transcripts were obtained using featureCounts and used as input into DESeq2, within which normalization and differential gene expression analysis were performed. Wald test $P$ value statistics were calculated using DESeq2.

Retroviral transduction of the 4662 cell line with Tdt-Ova. The pMX-Tdt-Ova plasmid containing a pMXs retroviral backbone with full-length OVA fused to Td-Tomato was a gift from Li Lily Wang (Dartmouth College, New Hampshire, USA). Confluent HEK 293T packaging cells (70\%-90\%) were transfected with the plasmid and a packaging mix (pCMV-Gag/Pol and pVSV-G) using lipofectamine to produce viral supernatant. 4662 cells were plated in a 24 -well plate until reaching 50\% confluence. Cells were washed, and $1 \mathrm{ml}$ of a 1:2 virus/DMEM solution was incubated for 36 to 48 hours (diluted at 24 hours with an additional $\mathrm{ml}$ of DMEM). Cells were passaged into larger flasks and sorted by flow cytometry for the top $15 \%$ of Td-Tomato-expressing cells to create an enriched cell line. Cells were then single cell sorted into a 96-well plate to create single-cell clones of TdT-Ova4662. 
IFN- $\gamma$ stimulation assay. To assess for processing and presentation of SIINFEKL peptide on MHC class I under conditions of IFN- $\gamma$ stimulation, 4662 parental Tdt-Ova-transduced clones (V6, G7, and G10 single-cell clones) were plated in T25 flasks, and at $50 \%$ confluence, 500 units/ $\mu 1$ of IFN- $\gamma$ (R\&D systems) was added to one flask of each cell line and incubated for 24 hours, while a duplicate flask remained unstimulated. As a positive control, SIINFEKL peptide was then added at $10 \mu \mathrm{g} / \mathrm{ml}$ for 30 minutes at $37^{\circ} \mathrm{C}$ to stimulated and unstimulated 4662 cells. Cells were then trypsinized, washed, and stained for viability and Ova-H2-K $\mathrm{K}^{\mathrm{b}}$ by flow cytometry.

Flow cytometry and tetramer staining. Following preparation of a single-cell suspension, up to $5 \times 10^{6}$ cells were plated per well in a 96-well plate, washed, and stained with fluorochrome-labeled antibodies at $4^{\circ} \mathrm{C}$ for 20 minutes in a buffer of PBS/1\% FCS and 0.5 mM EDTA. For tetramer stains, prior to surface staining, cells were incubated at $37^{\circ} \mathrm{C}$ with positive or negative tetramer at 1:100 in FACS buffer and then diluted with a $2 \times$ concentrate of the remaining surface stains. Cell were run on a FACSCanto flow cytometer (BD Biosciences) and analyzed with Flowjo software.

Intracellular cytokine stimulation assay. A single-cell suspension was prepared of tumor and spleen and samples were plated in duplicate in a 96-well plate for stimulated and unstimulated treatments. Stimulation was performed using RPMI supplemented with L-glutamine, gentamicin, 10\% FCS, and 0.05 mM 2-ME. For unstimulated samples, GolgiStop (Monensin) was added to media at 1:150, and stimulated samples were additionally incubated with PMA (1 mg/ml stock) at 1:20,000 and ionomycin (1 mM stock) at 1:100. Both stimulated and unstimulated samples were incubated for 5 hours at $37^{\circ} \mathrm{C}$. Viability was then assessed using the Live/Dead Fixable Aqua Dead Cell Stain Kit, followed by surface staining and fixation/permeabilization for intracellular staining.

Statistics. Variations between two groups were determined by an unpaired 2-tailed Student's $t$ test. Differences between three (or more) groups for one factor were analyzed by 1-way ANOVA, with Tukey's multiple comparison test used as a post-hoc test to evaluate differences between any two groups. To study the effect of multiple factors across multiple groups, 2-way ANOVA was utilized with the Sidak's multiple comparison test for post-hoc evaluation of differences between any two groups. Tumor growth curves were analyzed by 2-way ANOVA, with Tukey's multiple comparisons of means used as a post-hoc test to determine differences between any two groups. Survival curves were assessed by the log-rank (Mantel-Cox) test. All statistical analyses were performed using GraphPad Prism 6 (GraphPad), except 2-way ANOVA and related post-hoc testing, which were performed on $\mathrm{R}$ Statistical Software (R Core Team). $P \leq 0.05$ indicates differences that are statistically significant.

Study approval. These studies in animals were reviewed and approved by the University of Pennsylvania Institutional Animal Care and Use Committee.

\section{Author contributions}

RAE and RHV conceived and designed the study. RAE, MSD, AJR, TC, MWR, DLB, KTB, NM, and RHV developed methodology. RAE, MSD, AJR, TC, JHL, and NM acquired data. RAE, MSD, AJR, TC, JHL, BZS, JLR, NM, RW, and RHV analyzed and interpreted data. RAE, MSD, AJR, TC, MWR, JHL, DLB, KTB, BZS, JLR, NM, RW, and RHV wrote, reviewed, and/or revised the manuscript. RHV supervised the study.

\section{Acknowledgments}

The authors thank Cynthia Clendenin for management of the mouse colony and Jose Conejo-Garcia, Brian Keith, Nicola Mason, Kate Nathanson, Golnaz Vahedi, Lili Wang, and John Wherry for helpful discussions. This work was supported by NIH grants R01 CA169123 (RHV and BZS), T32 HL007439 (MSD), and T32 CA009140 (RW); the Parker Institute for Cancer Immunotherapy (RHV); and a grant from the American Cancer Society (KTB).

Address correspondence to: Robert H. Vonderheide, Perelman School of Medicine at the University of Pennsylvania, 3400 Civic Center Blvd., Philadelphia, Pennsylvania 19104, USA. Phone: 215.746.8901; E-mail: rhv@exchange.upenn.edu.

1. Sharma P, Allison JP. The future of immune checkpoint therapy. Science. 2015;348(6230):56-61. 
2. Shankaran V, et al. IFNgamma and lymphocytes prevent primary tumour development and shape tumour immunogenicity. Nature. 2001;410(6832):1107-1111.

3. Matsushita $\mathrm{H}$, et al. Cancer exome analysis reveals a T-cell-dependent mechanism of cancer immunoediting. Nature 2012;482(7385):400-404.

4. Zhang B, et al. Immune surveillance and therapy of lymphomas driven by Epstein-Barr virus protein LMP1 in a mouse model. Cell. 2012;148(4):739-751.

5. Willimsky G, Blankenstein T. Sporadic immunogenic tumours avoid destruction by inducing T-cell tolerance. Nature. 2005;437(7055):141-146.

6. Casanovas O, Hicklin DJ, Bergers G, Hanahan D. Drug resistance by evasion of antiangiogenic targeting of VEGF signaling in late-stage pancreatic islet tumors. Cancer Cell. 2005;8(4):299-309.

7. DeNardo DG, et al. CD4(+) T cells regulate pulmonary metastasis of mammary carcinomas by enhancing protumor properties of macrophages. Cancer Cell. 2009;16(2):91-102.

8. Ciampricotti M, et al. Development of metastatic HER2(+) breast cancer is independent of the adaptive immune system. $J$ Pathol. 2011;224(1):56-66

9. Ciampricotti M, Hau CS, Doornebal CW, Jonkers J, de Visser KE. Chemotherapy response of spontaneous mammary tumors is independent of the adaptive immune system. Nat Med. 2012;18(3):344-6; author reply 346.

10. Vonderheide RH, Bayne LJ. Inflammatory networks and immune surveillance of pancreatic carcinoma. Curr Opin Immunol. 2013;25(2):200-205

11. Joyce JA, Fearon DT. T cell exclusion, immune privilege, and the tumor microenvironment. Science. 2015;348(6230):74-80.

12. Clark CE, Hingorani SR, Mick R, Combs C, Tuveson DA, Vonderheide RH. Dynamics of the immune reaction to pancreatic cancer from inception to invasion. Cancer Res. 2007;67(19):9518-9527.

13. Clark CE, Beatty GL, Vonderheide RH. Immunosurveillance of pancreatic adenocarcinoma: insights from genetically engineered mouse models of cancer. Cancer Lett. 2009;279(1):1-7.

14. Hingorani SR, et al. Trp53R172H and KrasG12D cooperate to promote chromosomal instability and widely metastatic pancreatic ductal adenocarcinoma in mice. Cancer Cell. 2005;7(5):469-483.

15. Rahib L, Smith BD, Aizenberg R, Rosenzweig AB, Fleshman JM, Matrisian LM. Projecting cancer incidence and deaths to 2030: the unexpected burden of thyroid, liver, and pancreas cancers in the United States. Cancer Res. 2014;74(11):2913-2921

16. Winograd R, et al. Induction of T-cell Immunity Overcomes Complete Resistance to PD-1 and CTLA-4 Blockade and Improves Survival in Pancreatic Carcinoma. Cancer Immunol Res. 2015;3(4):399-411.

17. Feig C, et al. Targeting CXCL12 from FAP-expressing carcinoma-associated fibroblasts synergizes with anti-PD-L1 immunotherapy in pancreatic cancer. Proc Natl Acad Sci U S A. 2013;110(50):20212-20217.

18. Beatty GL, et al. CD40 agonists alter tumor stroma and show efficacy against pancreatic carcinoma in mice and humans. Science. 2011;331(6024):1612-1616.

19. Akbay EA, et al. Activation of the PD-1 pathway contributes to immune escape in EGFR-driven lung tumors. Cancer Discov. 2013;3(12):1355-1363

20. Skoulidis F, et al. Co-occurring genomic alterations define major subsets of KRAS-mutant lung adenocarcinoma with distinct biology, immune profiles, and therapeutic vulnerabilities. Cancer Discov. 2015;5(8):860-877.

21. Bailey P, et al. Genomic analyses identify molecular subtypes of pancreatic cancer. Nature. 2016;531(7592):47-52.

22. DuPage M, Mazumdar C, Schmidt LM, Cheung AF, Jacks T. Expression of tumour-specific antigens underlies cancer immunoediting. Nature. 2012;482(7385):405-409.

23. Lo A, et al. Tumor-promoting desmoplasia is disrupted by depleting FAP-expressing stromal cells. Cancer Res. 2015;75(14):2800-2810.

24. Gubin MM, et al. Checkpoint blockade cancer immunotherapy targets tumour-specific mutant antigens. Nature. 2014;515(7528):577-581.

25. DuPage M, et al. Endogenous T cell responses to antigens expressed in lung adenocarcinomas delay malignant tumor progression. Cancer Cell. 2011;19(1):72-85.

26. Spranger S, Bao R, Gajewski TF. Melanoma-intrinsic $\beta$-catenin signalling prevents anti-tumour immunity. Nature. 2015;523(7559):231-235.

27. Wölfel T, et al. A p16INK4a-insensitive CDK4 mutant targeted by cytolytic T lymphocytes in a human melanoma. Science. 1995;269(5228):1281-1284.

28. Robbins PF, et al. A mutated beta-catenin gene encodes a melanoma-specific antigen recognized by tumor infiltrating lymphocytes. J Exp Med. 1996;183(3):1185-1192.

29. Dubey $\mathrm{P}$, et al. The immunodominant antigen of an ultraviolet-induced regressor tumor is generated by a somatic point mutation in the DEAD box helicase p68. J Exp Med. 1997;185(4):695-705.

30. Westcott PM, et al. The mutational landscapes of genetic and chemical models of Kras-driven lung cancer. Nature. 2015;517(7535):489-492.

31. Jones $\mathrm{S}$, et al. Core signaling pathways in human pancreatic cancers revealed by global genomic analyses. Science. 2008;321(5897):1801-1806.

32. Waddell N, et al. Whole genomes redefine the mutational landscape of pancreatic cancer. Nature. 2015;518(7540):495-501.

33. Sausen M, et al. Clinical implications of genomic alterations in the tumour and circulation of pancreatic cancer patients. Nat Commun. 2015;6:7686

34. Castle JC, et al. Exploiting the mutanome for tumor vaccination. Cancer Res. 2012;72(5):1081-1091.

35. Wang L, Pino-Lagos K, de Vries VC, Guleria I, Sayegh MH, Noelle RJ. Programmed death 1 ligand signaling regulates the generation of adaptive Foxp3+CD4+ regulatory T cells. Proc Natl Acad Sci U S A. 2008;105(27):9331-9336.

36. Rötzschke $\mathrm{O}$, et al. Isolation and analysis of naturally processed viral peptides as recognized by cytotoxic T cells. Nature. 1990;348(6298):252-254.

37. Ehst BD, Ingulli E, Jenkins MK. Development of a novel transgenic mouse for the study of interactions between CD4 and CD8 T cells during graft rejection. Am J Transplant. 2003;3(11):1355-1362. 
38. Wherry EJ, Kurachi M. Molecular and cellular insights into T cell exhaustion. Nat Rev Immunol. 2015;15(8):486-499.

39. Bayne LJ, et al. Tumor-derived granulocyte-macrophage colony-stimulating factor regulates myeloid inflammation and $\mathrm{T}$ cell immunity in pancreatic cancer. Cancer Cell. 2012;21(6):822-835.

40. Pylayeva-Gupta Y, Lee KE, Hajdu CH, Miller G, Bar-Sagi D. Oncogenic Kras-induced GM-CSF production promotes the development of pancreatic neoplasia. Cancer Cell. 2012;21(6):836-847.

41. Gunderson AJ, et al. Bruton tyrosine kinase-dependent immune cell cross-talk drives pancreas cancer. Cancer Discov. 2016;6(3):270-285

42. Ryan SO, Gantt KR, Finn OJ. Tumor antigen-based immunotherapy and immunoprevention of cancer. Int Arch Allergy Immunol. 2007;142(3):179-189.

43. Cobbold M, et al. MHC class I-associated phosphopeptides are the targets of memory-like immunity in leukemia. Sci Transl Med. 2013;5(203):203ra125.

44. Foley K, et al. Current progress in immunotherapy for pancreatic cancer [published online ahead of print December 23, 2015] Cancer Lett. doi: 10.1016/j.canlet.2015.12.020.

45. Byrne KT, Vonderheide RH. CD40 stimulation obviates innate sensors and drives T cell immunity in cancer. Cell Rep. 2016;15(12):2719-2732.

46. Cibulskis K, et al. Sensitive detection of somatic point mutations in impure and heterogeneous cancer samples. Nat Biotechnol. $2013 ; 31(3): 213-219$ 